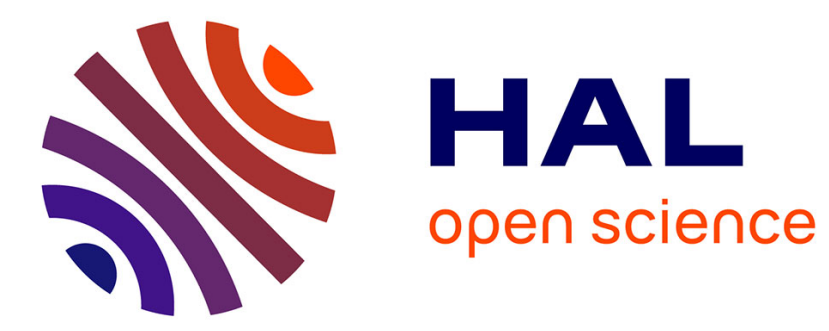

\title{
Quantification of the influence of the track geometry variability on the train dynamics
}

Guillaume Perrin, Denis Duhamel, Christian Soize, Christine Funfschilling

\section{To cite this version:}

Guillaume Perrin, Denis Duhamel, Christian Soize, Christine Funfschilling. Quantification of the influence of the track geometry variability on the train dynamics. Mechanical Systems and Signal Processing, 2015, 60-61, pp.945-957. 10.1016/j.ymssp.2015.01.004 . hal-01137530

\section{HAL Id: hal-01137530 \\ https://hal.science/hal-01137530}

Submitted on 30 Mar 2015

HAL is a multi-disciplinary open access archive for the deposit and dissemination of scientific research documents, whether they are published or not. The documents may come from teaching and research institutions in France or abroad, or from public or private research centers.
L'archive ouverte pluridisciplinaire HAL, est destinée au dépôt et à la diffusion de documents scientifiques de niveau recherche, publiés ou non, émanant des établissements d'enseignement et de recherche français ou étrangers, des laboratoires publics ou privés. 


\title{
Quantification of the influence of the track geometry variability on the train dynamics.
}

\author{
G. Perrin ${ }^{\mathrm{a}, \mathrm{b}, \mathrm{c}, *}$, D. Duhamel ${ }^{\mathrm{a}}$, C. Soize ${ }^{\mathrm{b}}$, C. Funfschilling ${ }^{\mathrm{c}}$ \\ ${ }^{a}$ Université Paris-Est, Navier (UMR 8205 ENPC-IFSTTAR-CNRS), Ecole Nationale des Ponts et \\ Chaussées, 6 et 8 Avenue Blaise Pascal, Cité Descartes, Champs sur Marne, 77455 Marne-la-Vallée, Cedex \\ 2, France. \\ ${ }^{b}$ Université Paris-Est, Modélisation et Simulation Multi-Échelle (MSME UMR 8208 CNRS), 5 Bd. \\ Descartes, 77454 Marne-la-Vallée, France. \\ ${ }^{c}$ SNCF, Innovation and Research Department, Immeuble Lumière, 40 avenue des Terroirs de France, \\ 75611, Paris, Cedex 12, France.
}

\begin{abstract}
In a context of increasing interoperability, several high speed trains, such as ICE, TGV, ETR 500, are likely to run on the same tracks, whereas they have been originally designed for specific and different railway networks. Due to different mechanical properties and structures, the dynamic behaviors, the aggressiveness of the vehicle on the track and the probabilities of exceeding security and comfort thresholds will be very different from one train to an other. These maintenance, certification and comfort criteria depend on the dynamic interaction between the vehicle and the railway track and in particularly on the contact loads between the wheels and the rail, which are very hard to evaluate experimentally. Moreover, the trackvehicle system being strongly non-linear, this dynamic interaction has to be analyzed not only on a few track portions but on the whole realm of possibilities of running conditions that the train is bound to be confronted to during its life cycle.

The idea of this paper is therefore to show to what extent this influence of the track geometry variability on the train dynamics can be analyzed from the coupling of a deterministic multibody modeling of the train with a track geometry stochastic modeling, which has been identified and validated from experimental data.
\end{abstract}

Keywords:

Railway track geometry, random fields, train dynamics, risk assessment.

\section{Introduction}

To face the always more demanding challenges of the railway field, the expected benefits of simulation versus experiment are multiple, as it would allow cheaper, shorter, and more

\footnotetext{
*Corresponding author

Email addresses: guillaume.perrin@enpc.fr (G. Perrin), denis.duhamel@enpc.fr (D. Duhamel), christian.soize@univ-paris-est.fr (C. Soize), christine.funfschilling@sncf.fr (C. Funfschilling)
} 

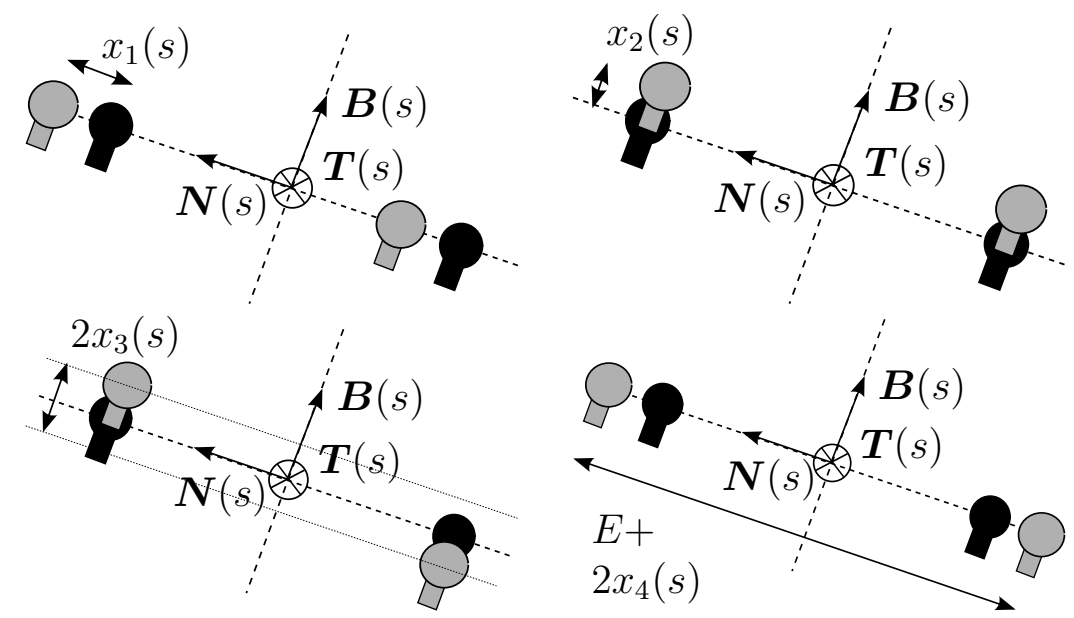

Figure 1: Parametrization of the track irregularities, where $E$ the theoretical track gauge, $s$ is the curvilinear abscissa of the track and $\left(\boldsymbol{O}_{\mathrm{NT}}(s), \mathbf{T}(s), \mathbf{N}(s), \mathbf{B}(s)\right)$ is the Frenet frame (for each rail, the mean position is represented in black, whereas the real position is in grey).

practical procedures. However, if simulation has to be used in security, maintenance and comfort prospects, it has to be representative of the physical behavior of the track-vehicle system. The numerical models of the train and of the wheel-rail contact have indeed to be fully validated, and a procedure to accurately investigate the diversity of running conditions that the train can be confronted to during its life cycle has to be defined. Such running conditions refer, in particular, to a double scale description of the track geometry. Indeed, whereas the mean line position of the perfect track, which is chosen at the building of a new track, is characterized by three curvilinear quantities, which are the vertical curvature $c_{V}$, the horizontal curvature $c_{H}$, and the track superelevation $c_{L}$, the description of four kinds of track irregularities has to be added to define the actual positions of the two rails, which are the lateral and vertical alignment irregularities $x_{1}$ and $x_{2}$, the cant deficiencies $x_{3}$ and the gauge irregularities $x_{4}$ (see Figure 1). These four track irregularities, which are the main source of excitation of the train dynamics, are moreover in constant evolution, which is due to the train dynamics, to the modifications of the track substructure and to maintenance operations. Numerical methods to quantify the influence of this track irregularity variability on the train dynamics are therefore needed.

The general scheme for probabilistic analysis is usually divided in three steps [1, 2, 3]. First, the mechanical model and the associated input parameters and output criteria have to be defined precisely. Three kinds of inputs are needed for a railway model to be defined: the vehicle model, the track model, and the wheel-rail contact model. Given these three inputs, the train response can be computed as the solution of a system of coupled equations that are strongly nonlinear. Once these equations have been solved, the spatial accelerations of each mass body, as well as the internal and external loads are available. These railway outputs can then be post-processed to define safety, comfort and maintenance criteria. Hence, thanks to the coupling of a series of breakthroughs in the modeling of complex mechanical systems 
[4, 5, 6, 7, 8, 9, 10, 11, 12, 13] with an increase of the computational resources, it is now possible to compare the simulated dynamics of a real train on a measured track geometry and the measured dynamics of the same train on the same track [14]. Then, much attention has to be paid to the modeling of the input variability, as any error on the input will be propagated to the output. Only the uncertainty in the track irregularities is addressed in this work. Finally, the variability has to be propagated through the mechanical model. Noticing that the railway mechanical system is based on a very high number of variable input parameters, that the train response is very sensitive, very non linear, and very fuzzy with respect to these input parameters, it appears that the best method to propagate the track variability to the train dynamics is the Monte Carlo (MC) method [15]. If such a MC method is used, a method to generate independent realistic and representative track conditions is thus needed. In this prospect, methods based on the one-sided power spectral density (PSD) functions of the track geometry [16, 17, 18] could be introduced to statistically characterize the random irregularities. From a time-frequency transformation based on a spectral representation [19, 20], track irregularities can indeed be generated from these PSD functions, which can be either estimated from measured track irregularities or from track safety standards such as the ones given by the Federal Railroad Administration [21]. In that case, the railway track irregularities are however seen as four independent Gaussian random fields that are assumed to be ergodic and stationary in space. These track irregularities are nevertheless strongly dependent in practice, and due to the strong coupling of the train dynamics with the track degradation, they are actually neither stationary nor Gaussian. More recently, taking advantage of the recent developments in the modeling of non-Gaussian and non-stationary vector-valued random fields [22, 23, 24, 25, 26, 27, 28, 29, 30], an original construction of the track irregularities distribution has been proposed in [31]. Such a modeling allows then the generation of running conditions that are realistic from frequency and statistical points of view, and representative of the measured track quality.

Hence, the idea of this work is to show to what extent such a complex modeling of the track geometry can be coupled with a rigid-body modeling of a complete train to analyze the influence of the track irregularity variability on the train dynamics. To this end, Section 2 introduces first the railway stochastic modeling and its validation from experimental data. Section 3 underlines then how such a stochastic modeling of the railway system can be used to perform robust comparisons between trains with different mechanical properties and to quantify the consequences of an increase of the train speed on the train stability and aggressiveness.

For confidentiality reasons, only qualitative analysis will be presented in this work as very few numerical values will be given.

\section{Railway stochastic modeling}

This section aims at presenting the numerical frame, on which the quantification of the influence of the track variability on the train dynamics is based. First, a description of the railway deterministic problem is introduced. Then, the railway stochastic modeling is 
described. The relevance of both deterministic and stochastic problems is moreover evaluated from comparisons to experimental data.

\subsection{Railway deterministic modeling}

\subsubsection{Definition of the deterministic modeling}

As presented in Section 1, a deterministic railway simulation is based on the introduction of three kinds of inputs.

- The vehicle model $\mathcal{V}$. Multibody simulations are usually employed to model the train dynamics. Carbodies, bogies and wheelsets are therefore modeled by rigid bodies linked with connections represented by rheologic models (dampers, springs, ...). For $1 \leq i \leq N_{\text {DoF }}$, and $t$ in $[0, T]$, we denote by $u_{i}(t)$ the position at time $t$ of the coordinate associated with each degree of freedom of the rigid body modeling of the train, and by $\dot{u}_{i}(t)=\frac{d u_{i}}{d t}(t)$ its time derivative. For instance, for a classical one-carriage TGV, which is made of 10 coaches, 13 bogies and 52 wheelsets that are linked by a series of suspensions and bumpstops, $N_{\text {DoF }}$ is about two hundreds (see Figure 2 for a simplified representation of the TGV).

- The track geometry, $\mathcal{T}$. As described in Section 1 , the track geometry refers to a double scale description. On the first hand, the track design gathers the horizontal curvature $c_{H}$, the vertical curvature $c_{V}$ and the cross level $c_{L}$, and corresponds to the description of a perfect track without irregularities. On the other hand, four track irregularities, $x_{1}, x_{2}, x_{3}$ and $x_{4}$, have been added to characterize the deviation of the real railway track toward this perfect track. It is moreover recalled that whereas the track design is chosen constant, such irregularities are in constant evolution.

- The contact model, $\mathcal{C}$, allows the computation of the contact forces between the rails and the wheels. In the railway community, these contact forces are almost always computed from the wheel profile and the rail profile thanks to the Hertz and Kalker theories [32, 33].

Introducing the vector of the generalized coordinates, at time $t$,

$$
\boldsymbol{U}(t)=\left(u_{1}(t), u_{2}(t), \ldots, \dot{u}_{1}(t), \dot{u}_{2}(t), \ldots\right),
$$

the train dynamics can therefore be determined by solving the Euler-Lagrange equation, which is written as:

$$
\frac{d}{d t}\left(\frac{\partial E_{c}}{\partial \dot{u}_{i}}\right)-\frac{\partial E_{c}}{\partial u_{i}}=L_{i}(\boldsymbol{U}, \mathcal{T}, \mathcal{C}), \quad 1 \leq i \leq N_{\mathrm{DoF}}
$$

with $E_{c}$ the total kinetic energy of the train, and $L_{i}(\boldsymbol{U}, \mathcal{T}, \mathcal{C})$ the general load that is applied to the degree of freedom $i$, which depends on the track geometry $\mathcal{T}$, on the wheel-rail contact $\mathcal{C}$ and on the generalized coordinated $\boldsymbol{U}$. Eq. (2) can be rewritten in a matricial form as: 


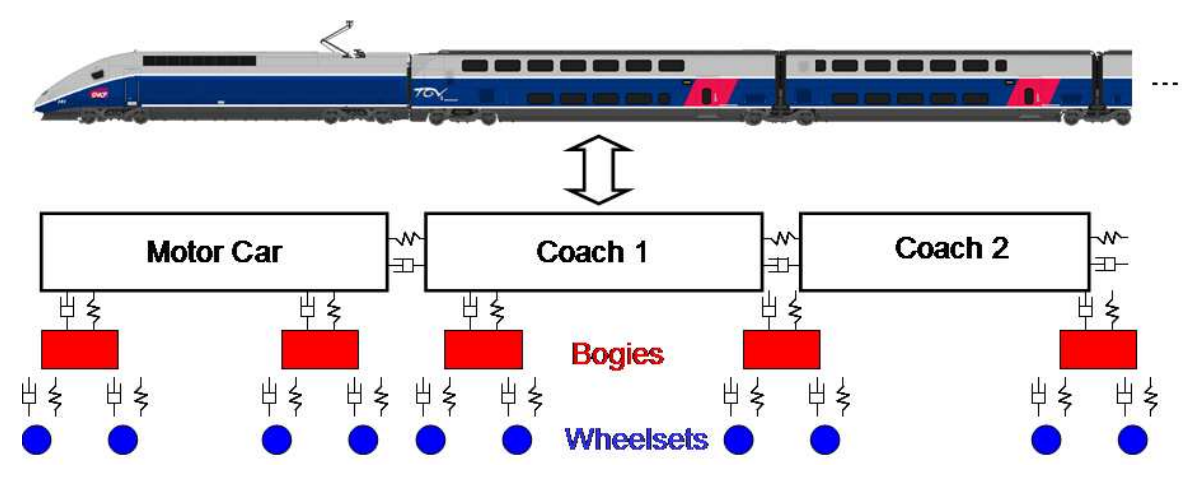

Figure 2: Simplified description of a multibody model of a TGV.

$$
[A(\boldsymbol{U})] \dot{\boldsymbol{U}}=\boldsymbol{F}(\boldsymbol{U}, \mathcal{T}, \mathcal{C})
$$

with $[A]$ and $\boldsymbol{F}$ two strongly nonlinear operators. This system is usually solved with an explicit time scheme. In the following, the commercial black-box software, Vampire (see [34, 35] for further details about this software), is used. The chosen time step of this explicit scheme was identified according to a convergence analysis and is generally taken equal to $10^{-4}$ second. The generalized coordinates vector $\boldsymbol{U}$ is then post-treated to define the final comfort and safety criteria associated with the railway system. In this work, five representative outputs are chosen to characterize the train dynamics, which can be classified in two categories.

1. First, the maximal values of the vertical and lateral accelerations in the train coaches, $\ddot{z}_{\text {max }}$ and $\ddot{y}_{\text {max }}$, are controlled to guarantee the comfort of the passengers.

2. Secondly, the safety and maintenance criteria of the track-vehicle system are based on the analysis of the wheel-rail contact forces. In this prospect, the following three criteria are generally introduced to characterize the vehicle dynamics on a given track geometry of total length $S^{\text {tot: }}$

- a shifting criterion:

$$
\left(Y_{\ell}+Y_{r}\right)_{\max }=\max _{\text {wheelset }}\left\{\max _{0 \leq s \leq S^{\text {tot }}}\left\{Y_{\ell}^{w}(s)+Y_{r}^{w}(s)\right\}\right\}
$$

- a derailment criterion:

$$
(Y / Q)_{\max }=\max _{\text {wheel } q}\left\{\max _{0 \leq s \leq S^{\text {tot }}}\left\{Y_{q}(s) / Q_{q}(s)\right\}\right\},
$$

- a wear criterion:

$$
(T \gamma)=\sum_{\text {wheel } q}\left\{\int_{0}^{S^{\text {tot }}} T_{q}(s) \gamma_{q}(s) d s\right\}
$$


where:

- $0 \leq s \leq S^{\text {tot }}$ is the curvilinear abscissa of the track;

- $Y_{\ell}^{w}$ and $Y_{r}^{w}$ are the left and right lateral forces of the same wheelset $w$, such that the higher $\left(Y_{\ell}+Y_{r}\right)_{\max }$, the more chance for a shifting of the track;

- $Y_{q}$ and $Q_{q}$ are the lateral and vertical components of the wheel-rail contact force at wheel $q$, such that the higher $(Y / Q)_{\max }$ is, the more on the flange a wheel of the train can be;

- $T_{q}$ and $\gamma_{q}$ are respectively the creep force and the slip at wheel $q$, such that the higher $(T \gamma)$, the higher the contact wear for one run of the complete train.

Finally, given a model of the wheel-rail contact $\mathcal{C}$, the deterministic railway problem corresponding to the dynamics of a vehicle $\mathcal{V}$ on a track geometry $\mathcal{T}$ can be expressed as:

$$
(\mathcal{V}, \mathcal{T}, \mathcal{C}) \mapsto \boldsymbol{c}=\boldsymbol{g}(\mathcal{V}, \mathcal{T}, \mathcal{C}), \quad \boldsymbol{c}=\left(\ddot{z}_{\max }, \ddot{y}_{\max },\left(Y_{\ell}+Y_{r}\right)_{\max },(Y / Q)_{\max },(T \gamma)\right)
$$

where it is reminded that $\boldsymbol{g}$ is a complex and nonlinear operator. These nonlinearities are mostly due to the train suspensions (especially the airsprings between the bogies and the coaches), to a series of bumpstops in the train description and to the wheel-rail contact forces. Moreover, due to the train dynamics, to the track irregularities and to the specific wheel and rail profiles, the contact positions between each wheel of the train and the rails keep changing. The wornest the track geometry is, the more discontinuous these changes are likely to be. For instance, the diversity of these contact positions and contact forces can be seen in Figure 4, which is based on the run of a train on a measured track geometry around a curve, whose design is shown in Figure 3.

\subsubsection{Domain of validity for the deterministic problem}

As a first comment on the validity of the deterministic railway modeling, it is important to point out that all European railway reference standards and reference maintenance guides only consider the low-frequency content, $f \leq f_{c}$, of the train dynamic quantities of interest (either simulated or measured). As presented in the former Section, the software Vampire is used to solve the railway deterministic problem. The train being constituted of rigid bodies, the simulated high-frequency response of the train cannot be physical. As an illustration, Figure 5 compares the measured and simulated frequency properties of a bogie of a TGV. As shown in Figure 6, although the transverse and vertical accelerations of the bogie are low-pass filtered at the reference cut-frequency $f=f_{c}$, it can be seen that the low-frequency response is well reproduced both in the time and frequency domains by the deterministic model. As a consequence, in agreement with the work achieved in [14], it is assumed that the proposed railway deterministic model is valid on the frequency band $0 \leq f \leq f_{c}$. In the following, each output of the train dynamics (whether measured or simulated) will thus be low-pass filtered at frequency $f_{c}$ before being analyzed. 


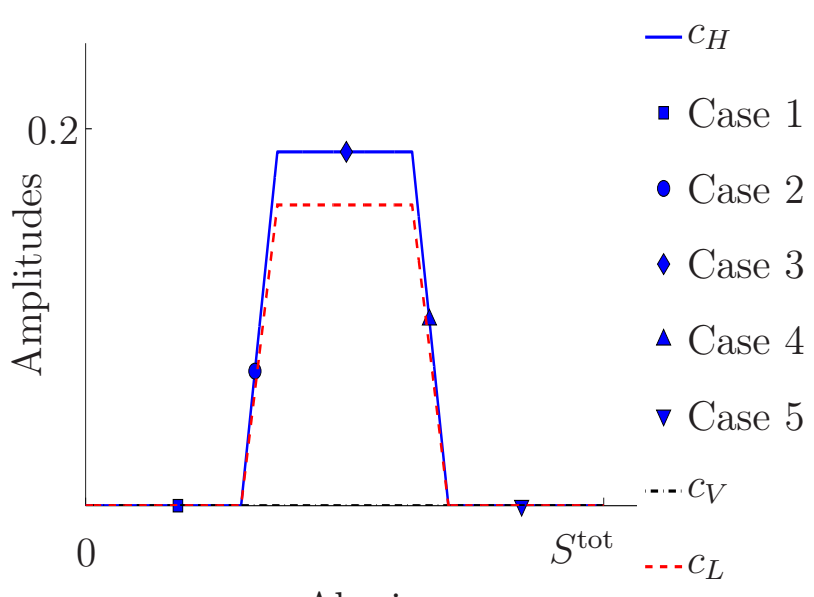

Abscissa $s$

Figure 3: Evolution of the horizontal curvature $c_{H}\left(\times \mathrm{km}^{-1}\right)$, the vertical curvature $c_{V}$ $\left(\times \mathrm{km}^{-1}\right)$ and of the cross level $c_{L}\left(\times \mathrm{m}^{-1}\right)$ with respect to the track curvilinear abscissa s.

\subsection{Railway stochastic problem}

\subsubsection{Definition of the stochastic problem}

As presented in Section 1, only the track geometry variability is analyzed in this work. The wheel and rail profiles of high speed trains and lines being checked and maintained very regularly, only perfect wheel and rail profiles will be considered in the following, such that the contact properties, $\mathcal{C}$, are chosen to be constant. It is moreover supposed that the track irregularities can be separated from the track design. Hence, in the following, the track design is also supposed to be constant, while the four track irregularities can vary. As a consequence, vector $\boldsymbol{c}$, which is defined by Eq. (17), becomes a random vector that is denoted by $\boldsymbol{C}=\left(C_{1}, C_{2}, C_{3}, C_{4}, C_{5}\right)$. It is reminded that by definition of vector $\boldsymbol{c}, C_{1}$ and $C_{2}$ refer to the vertical and lateral maximal accelerations in the train coaches, $C_{3}$ is the maximal value of the sum of the transverse loads of the wheelsets, $C_{4}$ is the maximal value of the $Y / Q$ ratio, and $C_{5}$ is the cumulated wear along the track. At last, given a fixed description of the track design, $\left(c_{H}, c_{V}, c_{L}\right)$, and a normalized model of train, $\mathcal{V}$, for which mechanical parameters are also fixed and have been accurately identified, the railway stochastic problem can be witten:

$$
\boldsymbol{X} \mapsto \boldsymbol{C}=\boldsymbol{G}\left(\boldsymbol{X} \mid c_{H}, c_{V}, c_{L}, \mathcal{V}, \mathcal{C}\right),
$$

where $\boldsymbol{X}=\left(X_{1}, X_{2}, X_{3}, X_{4}\right)$ is a non-Gaussian and non-stationary four-dimensional random field, which gathers the evolutions with respect to the curvilinear abscissa of the considered track design of the four track irregularities that were introduced in Section 1 . In this work, the distribution of $\boldsymbol{X}$ is moreover supposed to be identified according to the developments 
Case 1

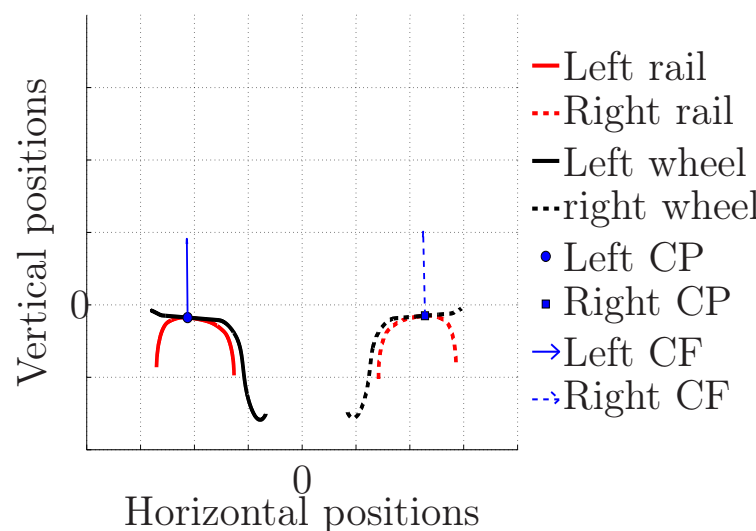

Case 3

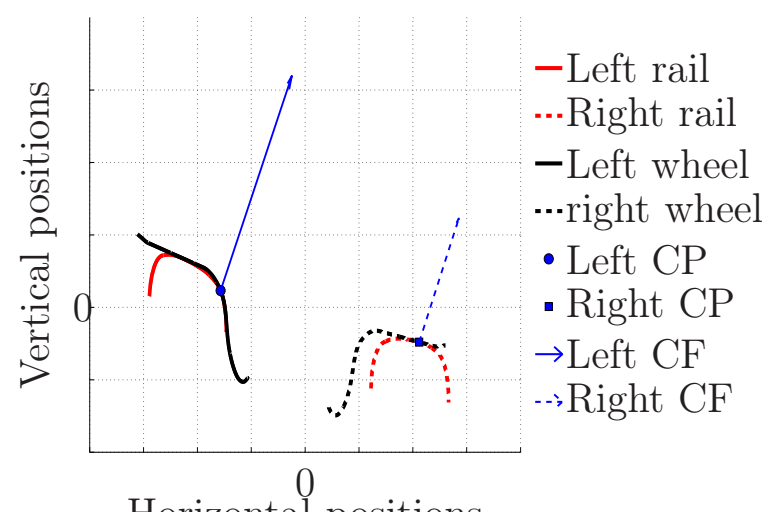

Horizontal positions

Case 5

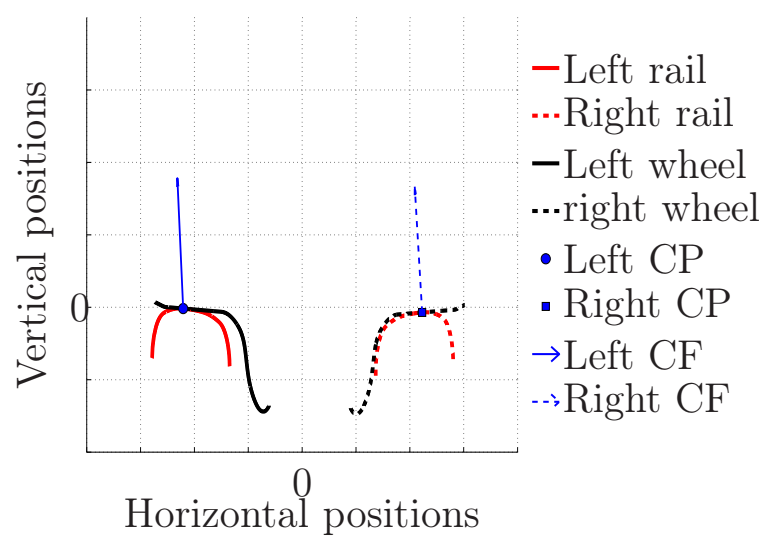

Case 2

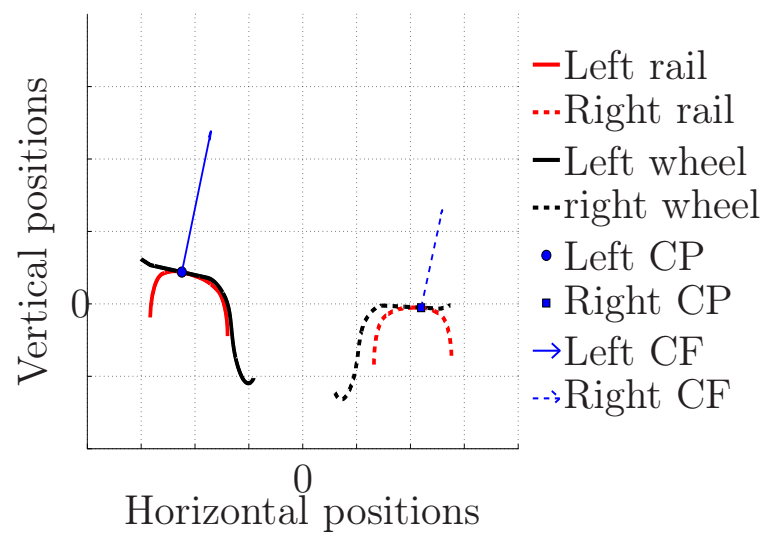

Case 4

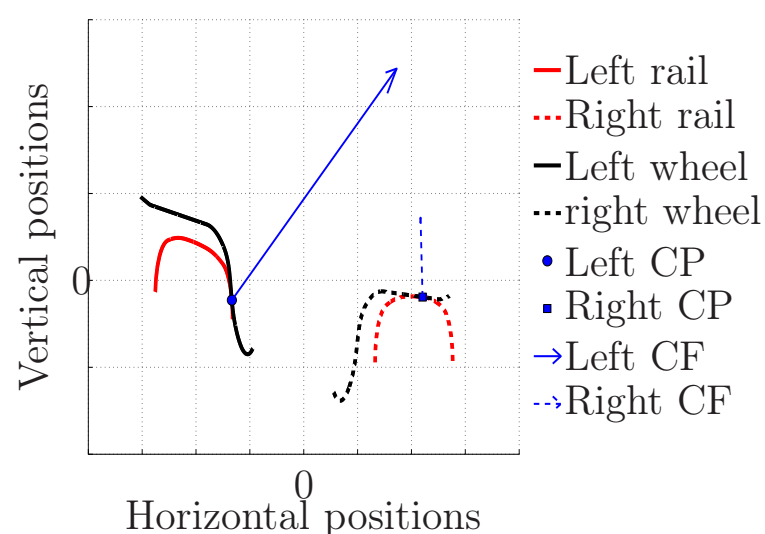

Figure 4: Evolution of the contact conditions with respect to the train dynamics and to the horizontal curvature $(\mathrm{CP}=$ contact point, $\mathrm{CF}=$ contact force). 


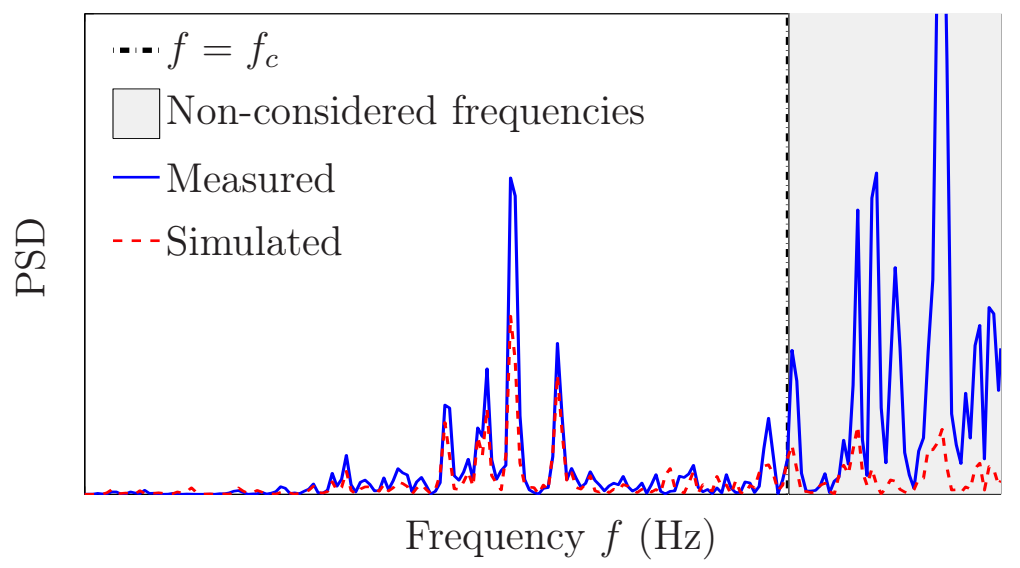

Figure 5: Frequency analysis of the transverse acceleration of the bogie of a TGV (figure extracted from [14], pp. 178-179).

Filtered vertical acceleration

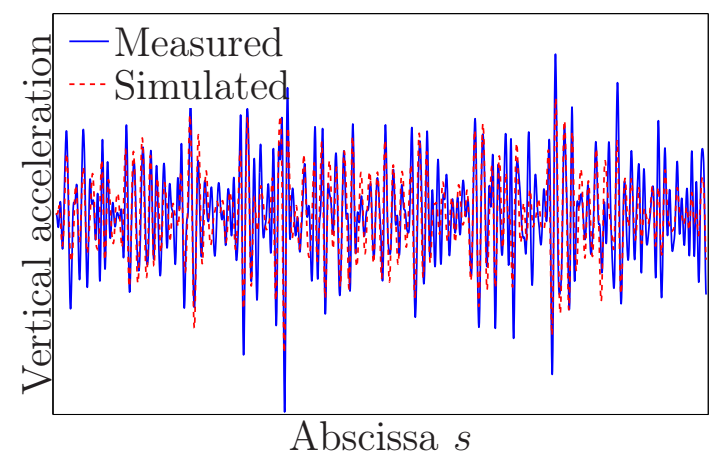

Filtered transverse accelerations

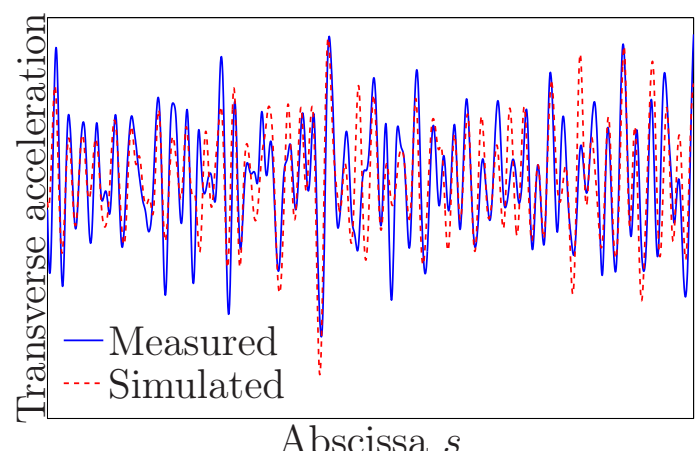

Figure 6: Validation of the low-frequency response of the deterministic model (figures extracted from [14], pp. 178-179). 
achieved in [31], from the measurement of the track geometry of a whole railway network. Hence, coupled with any track design, we admit that such a construction for $\boldsymbol{X}$ allows the generation of independent running conditions that are realistic and representative of the available measured track irregularities.

\subsubsection{Validation of the stochastic problem}

Two validations for the track geometry stochastic modeling, based on the train dynamics, are proposed in this section. In a first step, it is shown that the track generator coupled with the Vampire software allows the simulation of train accelerations that are similar to accelerations that have been recorded on a real high speed train on a real track. In a second step, another type of validation is proposed to evaluate the relevance of the track stochastic modeling for the analysis of the wheel-rail forces, as these forces can hardly be measured.

Relevance of the track stochastic modeling for the analysis of the train accelerations.

Since 2007, the measurement train IRIS-320 keeps monitoring the track geometry of the French high speed lines. The rigid body modeling of this train has therefore been achieved, simulations have then been performed at constant speed $\mathbb{S}$ on $\nu=500$ variable running conditions of total length $S^{\text {tot }}$. For each simulation, the track design is therefore chosen to be constant and to correspond once again to the one given by Figure 3 , whereas the track irregularities, which correspond to a particular realization, $\boldsymbol{X}\left(\Theta_{n}\right)$, of $\boldsymbol{X}$, are supposed to be different from one simulation to another. Hence, for all $s$ in $\left[0, S^{\text {tot }}\right]$, we respectively define $\widehat{C}_{z}^{\text {sim }}\left(\Theta_{n}, s\right)$ and $\widehat{C}_{y}^{\operatorname{sim}}\left(\Theta_{n}, s\right)$ as the vertical and lateral maximal values, at position $s$, of the accelerations in all the coaches of the train that is excited by the track irregularity $\boldsymbol{X}\left(\Theta_{n}\right)$. Given these two sets of train responses, $\left\{\widehat{C}_{z}^{\operatorname{sim}}\left(\Theta_{n}\right), 1 \leq n \leq \nu\right\}$ and $\left\{\widehat{C}_{y}^{\operatorname{sim}}\left(\Theta_{n}\right), 1 \leq n \leq \nu\right\}$, let $\left\{\mathcal{D}_{i}^{z}(s), s \in\left[0, S^{\text {tot }}\right], 1 \leq i \leq 10\right\}$ and $\left\{\mathcal{D}_{i}^{y}(s), s \in\left[0, S^{\text {tot }}\right], 1 \leq i \leq 10\right\}$ be the decile functions, such that at each position $s, i / 10$ of the values of $\widehat{C}_{z}^{\operatorname{sim}}\left(\Theta_{n}, s\right)$ and $\widehat{C}_{y}^{\operatorname{sim}}\left(\Theta_{n}, s\right)$ are in $\mathcal{D}_{i}^{z}(s)$ and $\mathcal{D}_{i}^{y}(s)$ respectively. These decile functions, whose representations are shown in Figure 7, allow us to evaluate the influence of the track irregularity variability on such maxima.

The IRIS-320 train is moreover equipped with accelerometers that record the vertical and transverse accelerations at three coaches,

$$
\left\{\ddot{y}_{C}^{(1)}, \ddot{y}_{C}^{(2)}, \ddot{y}_{C}^{(3)}, \ddot{z}_{C}^{(1)}, \ddot{z}_{C}^{(2)}, \ddot{z}_{C}^{(3)}\right\} .
$$

In order to evaluate the relevance of the former results for the maximal accelerations in the train coaches, we define $\widehat{C}_{z}^{\exp }$ and $\widehat{C}_{y}^{\exp }$, such that for any value of the curvilinear abscissa of the track, $s$, we get:

$$
\begin{aligned}
& \widehat{C}_{z}^{\exp }(s)=\max _{i \in\{1,2,3\}}\left|\ddot{z}_{C}^{(i)}(s)\right|, \\
& \widehat{C}_{y}^{\exp }(s)=\max _{i \in\{1,2,3\}}\left|\ddot{y}_{C}^{(i)}(s)\right| .
\end{aligned}
$$




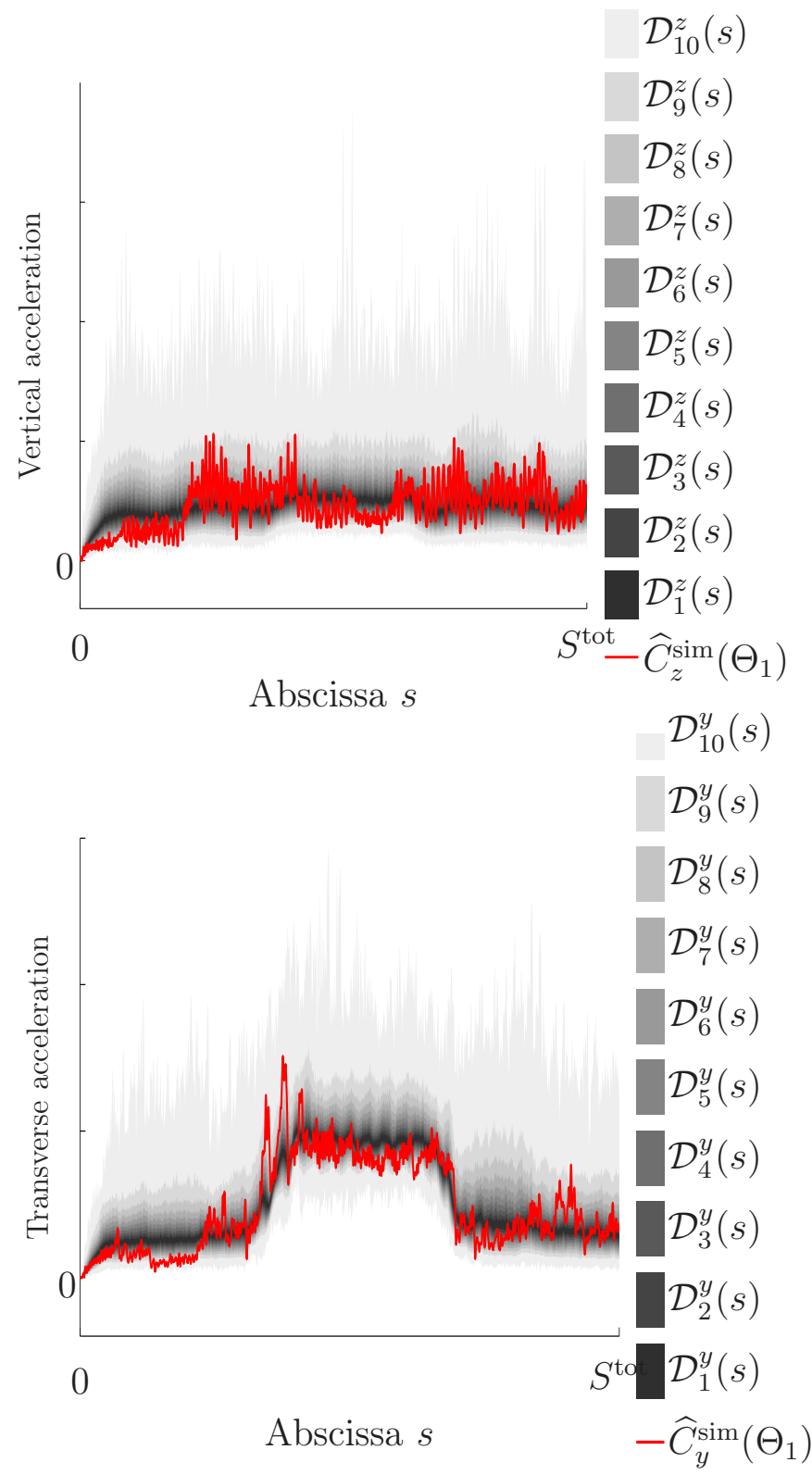

Figure 7: Influence of the track variability on the maximal values of the accelerations in the train coaches. 
Five particular evolutions for $\widehat{C}_{z}^{\exp }$ and $\widehat{C}_{y}^{\exp }$ over a length $S^{\text {tot }}$, which are denoted by $\left\{\widehat{C}_{z}^{\exp ,(1)}, \ldots, \widehat{C}_{z}^{\exp ,(5)}\right\}$ and $\left\{\widehat{C}_{y}^{\exp ,(1)}, \ldots, \widehat{C}_{y}^{\exp ,(5)}\right\}$, are then extracted from the experimental database collected by measurement train IRIS 320. These measurements were chosen as their dynamic characteristics were the most comparable to the simulated one, in terms of cross level, horizontal and vertical curvatures, speed of the train and length of the curve. If the chosen simulated dynamic characteristics were not similar to the extracted dynamic characteristics on the complete domain $\left[0, S^{\text {tot }}\right]$, non-valid domains were added to these figures. The evolutions of these measured accelerations are compared to the simulated ones in Figure 8. In the light of these results, the track generator coupled with the Vampire software seems to be able to simulate realistic and representative runs of the IRIS-320 train to analyze the link between the two first quantities of interest of the stochastic modeling, $C_{1}$ and $C_{2}$, and the track geometry variability.

Dynamic validation of the track generator for the analysis of the wheel-rail contact forces.

No on-track measurements of the contact forces between the train and the track at high speed being available, an other approach is proposed to evaluate the relevance of the track generator to simulate realistic and representative values for $C_{3}, C_{4}$ and $C_{5}$. To this end, the particular curve of total length $S^{\text {tot }}$ shown in Figure 3, is once again considered. From the available measurements of the track geometry, $\nu^{\exp }=400$ different track conditions of total length $S^{\text {tot }},\left\{\boldsymbol{X}_{\exp }\left(\theta_{1}\right), \ldots, \boldsymbol{X}_{\exp }\left(\theta_{\nu^{\exp }}\right)\right\}$, are gathered. The same normalized high-speed train $\mathcal{V}$, for which mechanical parameters are supposed to be accurately identified, is thus made run first on the $\nu^{\exp }$ measured track conditions, and then on the $\nu=500$ formerly generated track conditions, $\left\{\boldsymbol{X}\left(\Theta_{1}\right), \ldots, \boldsymbol{X}\left(\Theta_{\nu}\right)\right\}$, at the same speed $\mathbb{S}$. Eight quantities of interest that are representative of the train dynamics are then compared:

- the left and right transverse contact forces at the first wheelset of the first bogie of the motor car, $Q_{1}=Y_{M C}^{\ell}$ and $Q_{2}=Y_{M C}^{r}$;

- the left and right transverse contact forces at the second wheelset of the second bogie of the second passenger car, $Q_{3}=Y_{P C}^{\ell}$ and $Q_{4}=Y_{P C}^{r}$;

- the left and right $Y / Q$ ratio at the first wheelset of the first bogie of the motor car, $Q_{5}=(Y / Q)_{M C}^{\ell}$ and $Q_{6}=(Y / Q)_{M C}^{r}$;

- the left and right $Y / Q$ ratio at the second wheelset of the second bogie of the second passenger car, $Q_{7}=(Y / Q)_{P C}^{\ell}$ and $Q_{8}=(Y / Q)_{P C}^{r}$.

For $1 \leq i \leq 8$, we are now interested in the mean power spectral densities of $Q_{i}$ and the mean numbers of upcrossings of the level $u$ by $Q_{i}$ over the length $S^{\text {tot }}$, which are respectively denoted by $P S D^{\text {mes }}\left(Q_{i}\right)$ and $N_{\text {up }}^{\text {mes }}\left(Q_{i}, u, S^{\text {tot }}\right)$ when these quantities are computed from the measured track geometries and $P S D^{\text {gen }}\left(Q_{i}\right)$ and $N_{\mathrm{up}}^{\text {gen }}\left(Q_{i}, u, S^{\text {tot }}\right)$ when these quantities are computed from the generated track geometries. The comparisons between these quantities are represented in Figure 9, where a very good fit can be seen (for confidentiality reasons, 


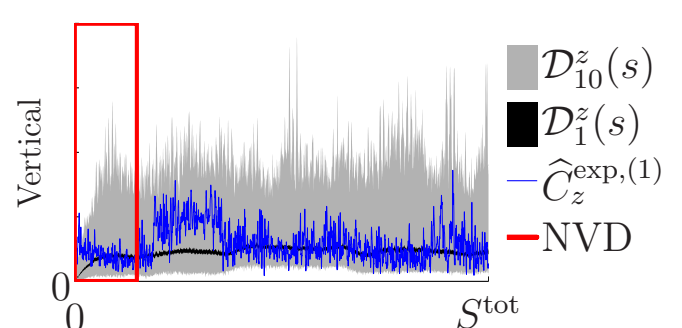

Abscissa $s$

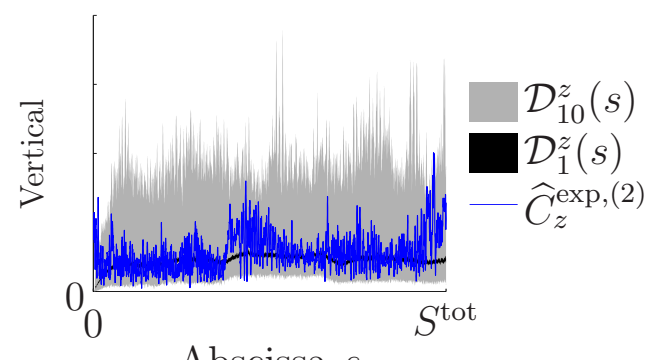

Abscissa $s$

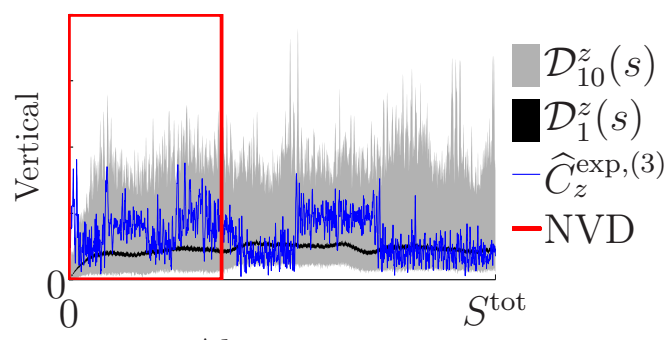

Abscissa $s$

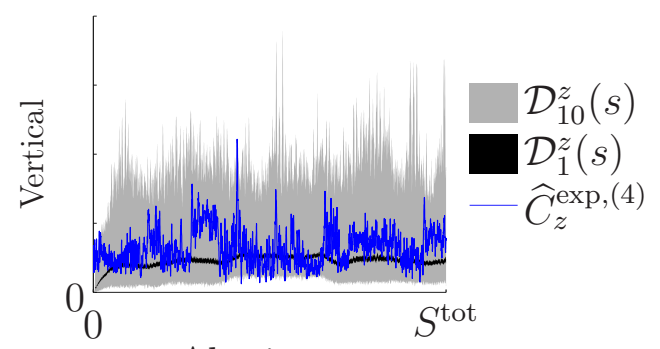

Abscissa $s$

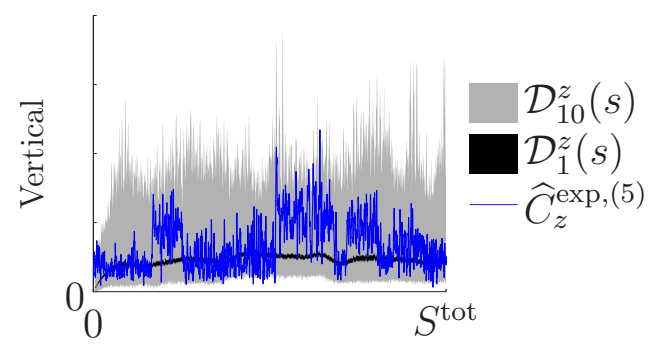

Abscissa $s$

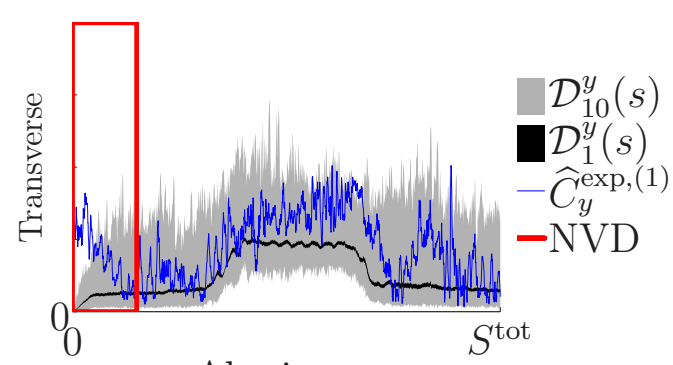

Abscissa $s$

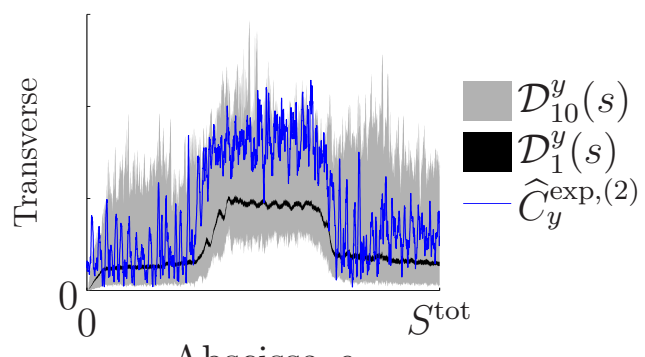

Abscissa $s$

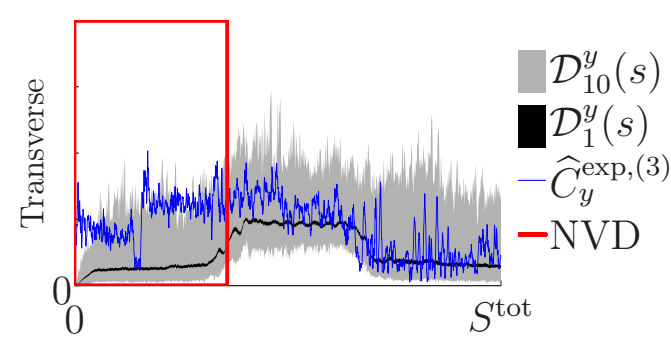

Abscissa $s$

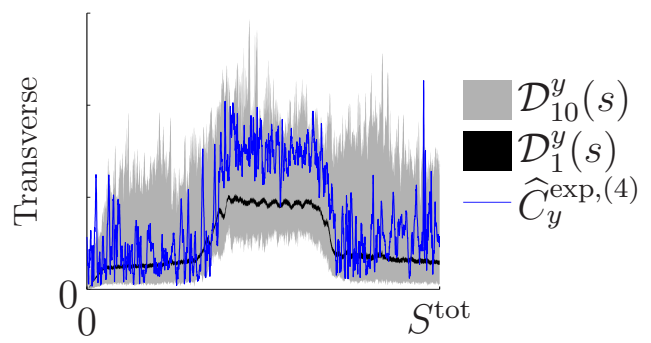

Abscissa $s$

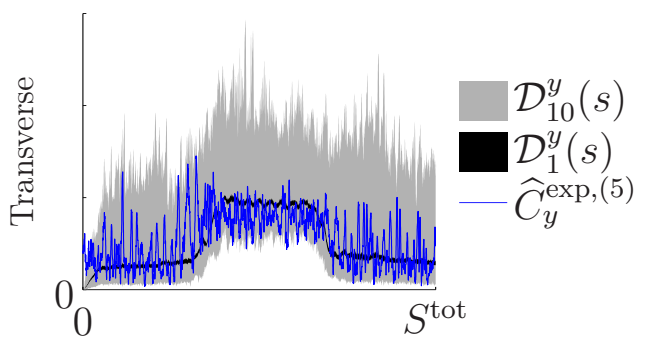

Abscissa $s$

Figure 8: Comparison between simulated and measured maximal values of the vertical and transverse accelerations in the train coaches (NVD $=$ non-valid domain). 
it is reminded that no numerical values for the abscissa of these graphs are given). The stochastic modeling of the track geometry, based on the developments carried out in [31], is thus relevant from the train response point of view. In the following, it is therefore supposed that the track stochastic modeling, coupled with the software Vampire is also relevant to investigate the relation between the track variability and the three quantities of interest $C_{3}$, $C_{4}$ and $C_{5}$.

\section{Influence of the track irregularities variability on the train dynamics}

As explained in Section 1, a better understanding of the specific link between the track irregularities and the train response is needed to optimize the maintenance, and to better anticipate the consequences of modifications of the running conditions. To this end, we denote by $P_{\boldsymbol{C}}(d \boldsymbol{x})=p_{C}(\boldsymbol{x}) d \boldsymbol{x}$ the multidimensional distribution of random vector $\boldsymbol{C}$, where $p_{C}$ is the associated density. This distribution is strongly related to the distribution of the track irregularity random field, $P_{\boldsymbol{X}}$ (see Eq. (8) ). Assuming that the latter distribution has been accurately identified, the track variability has now to be propagated through the railway model to characterize $P_{\boldsymbol{C}}$. As the statistical dimension of $\boldsymbol{X}$ is very high and as the relation between $P_{\boldsymbol{C}}$ and $P_{\boldsymbol{X}}$ is very complex and strongly nonlinear, the Monte Carlo method is chosen to evaluate $P_{\boldsymbol{C}}$. From $\nu$ independent realizations of $\boldsymbol{X},\left\{\boldsymbol{X}\left(\Theta_{1}\right), \ldots, \boldsymbol{X}\left(\Theta_{\nu}\right)\right\}$, which correspond to $\nu$ independent realistic and representative running conditions that a train can be confronted to during its lifecycle, $\nu$ independent realizations of $\boldsymbol{C},\left\{\boldsymbol{C}\left(\Theta_{1}\right), \ldots, \boldsymbol{C}\left(\Theta_{\nu}\right)\right\}$, can be deduced as:

$$
\boldsymbol{C}\left(\Theta_{n}\right)=\boldsymbol{G}\left(\boldsymbol{X}\left(\Theta_{n}\right) \mid c_{H}, c_{V}, c_{L}, \mathcal{V}, \mathcal{C}\right), \quad 1 \leq n \leq \nu
$$

The statistical properties of $\boldsymbol{C}$ are finally deduced from the analysis of this $\nu$-dimensional set of independent realizations of $\boldsymbol{C}$. Three applications of this stochastic modeling are now presented. These are based on the track design of total length $S^{\text {tot }}$ shown in Figure 3, and on $\nu=4,000$ independent running conditions. First, the compared influence of the track design and of the track irregularities is illustrated. Then, it is shown to what extent such a method can be used to quantify the influence of an increase of the train speed on $\boldsymbol{C}$, and to compare the safety and the aggressiveness of three different high speed trains.

\subsection{Influence of the track design}

The idea of this section is to quantify the importance of the track irregularities and of the track design on vector $\boldsymbol{C}$. In this prospect, the response of a normalized high train $\mathcal{V}$ to the former $\nu$ track conditions of total length $S^{\text {tot }}$ is analyzed. Four categories are considered: the alignment $(\mathrm{A})$, the curve entrance $(\mathrm{CE})$, the established curve $(\mathrm{C})$ and the curve exit (SC). The response of the train is therefore sorted with respect to these four curve categories, such that, for $1 \leq i \leq 5$, four values of the railway quantities of interest $C_{i}^{A}\left(\Theta_{n}\right), C_{i}^{E C}\left(\Theta_{n}\right)$, $C_{i}^{C}\left(\Theta_{n}\right)$ and $C_{i}^{S C}\left(\Theta_{n}\right)$ can be computed. Based on these sets of $\nu$ independent realizations, the PDFs of the components of $\boldsymbol{C}$ are estimated from a kernel smoothing method [2], and are represented in Figure 10, From these graphs, it can be seen that the influence of the 

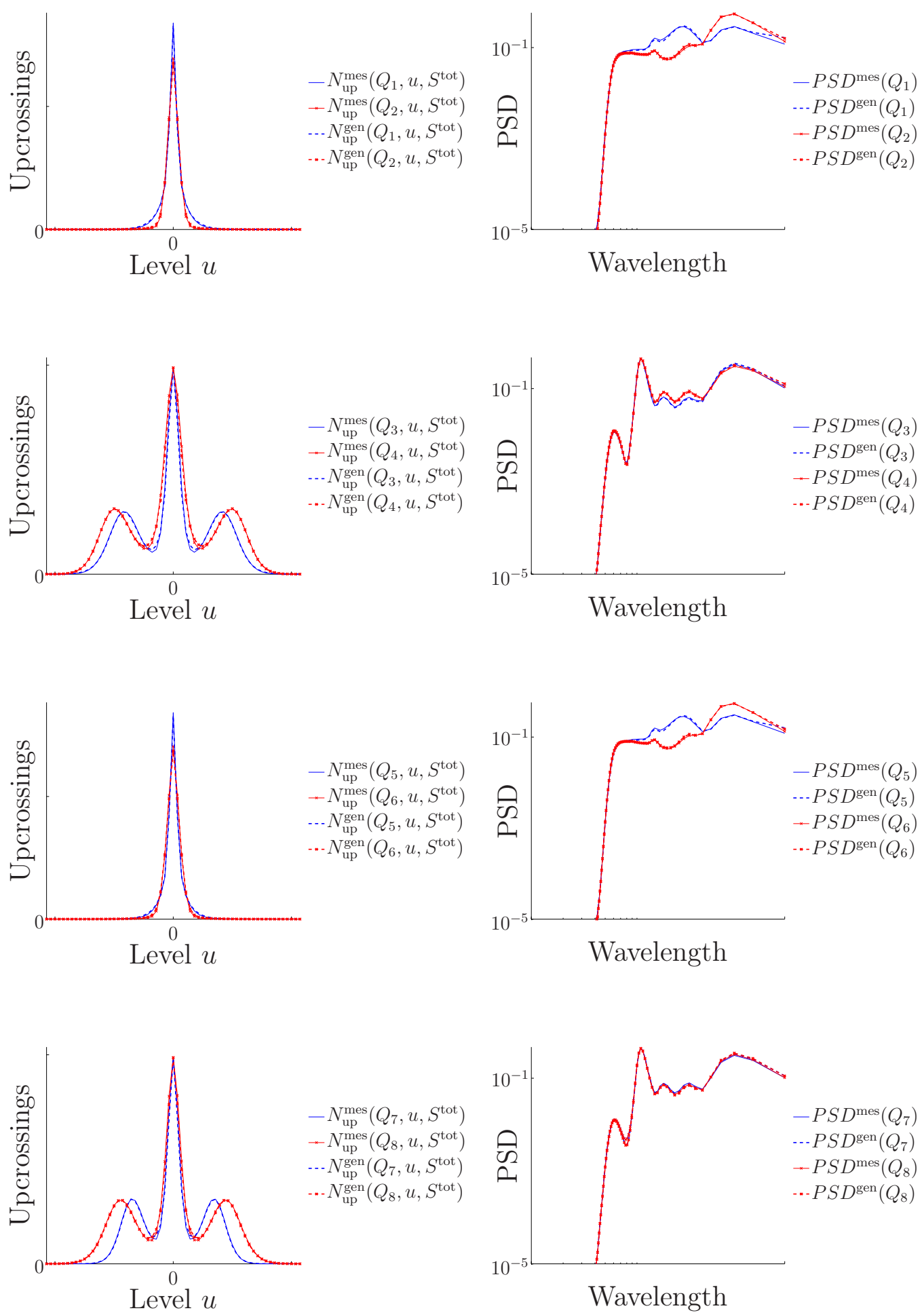

Figure 9: Spectral and statistical analysis of the dynamic quantities of interest $Q_{1}, Q_{2}, \ldots$, $Q_{8}$. 
track design on the wear criterion, $C_{5}$ is very high. The other dynamic quantities, $C_{1}, C_{2}$, $C_{3}$ and $C_{4}$ seem however to be much more dependent on the track irregularities than on the track design.

\subsection{Influence of an increase of the speed on the quantities of interest}

The second application deals with the influence of the train speed on the PDFs of the five considered criteria. Only the established curve configuration is shown. Railway simulations are therefore performed on the same $\nu$ realistic and representative track geometries, at the four speeds $\mathbb{S} 1=\mathbb{S}, \mathbb{S} 2=1.1 \mathbb{S}, \mathbb{S} 3=1.2 \mathcal{S}$ and $\mathbb{S} 4=1.3 \mathcal{S}$. Two other sets of simulations have then been carried out for a different value of the track superelevation, $c_{L}^{*}$, at speeds $\mathbb{S} 3=1.2 \mathbb{S}$ and $\mathbb{S} 4=1.3 \mathbb{S}$ in order to quantify the importance of this track design parameter with respect to the five studied criteria. In other words, whereas $c_{L}$ is chosen to compensate the train inertial acceleration in curve at speed $\mathbb{S} 1, c_{L}^{*}$ allows the compensation of the train inertial acceleration in curve at speed $\mathbb{S} 3$. For each speed, the PDFs of each component of $\boldsymbol{C}$ are once again estimated using a kernel smoothing method based on the $\nu=4,000$ independent railway simulations. These PDFs are represented in Figure 11. In this figure, the nonlinearity of the system can be noticed, as the consequences of an increase of the speed of $10 \%$ to $30 \%$ are much higher than $30 \%$ for each criterion. In particular, an increase of $30 \%$ of the speed of the train can yield an increase of more than $500 \%$ of the contact wear if the track superelevation is not adjusted. In addition, these figures emphasize the importance of the adjustment of the track superelevation to the speed, in terms of minization of the wear, of the shifting and of the risk of derailment.

\subsection{Comparison of three high speed trains}

In this section, it is supposed that three different models of three competitive high speed trains, $\mathcal{V}_{1}, \mathcal{V}_{2}$ and $\mathcal{V}_{2}$ are available. The mechanical parameters of these trains are very different and were carefully identified from experimental measurements. These three trains are thus made run on the same $\nu$ track geometries at the same speed $\mathbb{S}$. The PDFs of each criterion $C_{i}$ associated with each train are then shown in Figure 12. Hence, the stochastic modeling allows us to compare the dynamic response of these three trains when excited by a representative set of realistic track conditions. In particular, criteria $C_{3}$ and $C_{5}$ could be interesting indicators to compare the aggressiveness of each train.

\section{Conclusions and prospects}

A method to propagate the track geometry variability through railway mechanical simulations is nowadays of great interest to face always more challenging railway issues. In this prospect, this paper has presented a method based on the coupling of a track geometry stochastic modeling with a rigid-body modeling of a train. Based on experimental data, this stochastic modeling of the track geometry allows the generation of running conditions that are realistic and representative of the quality of the measured railway network. In this work, it has therefore been shown to what extent such a complex modeling, which takes into account the dependencies between the different track irregularities, can be used to analyze 

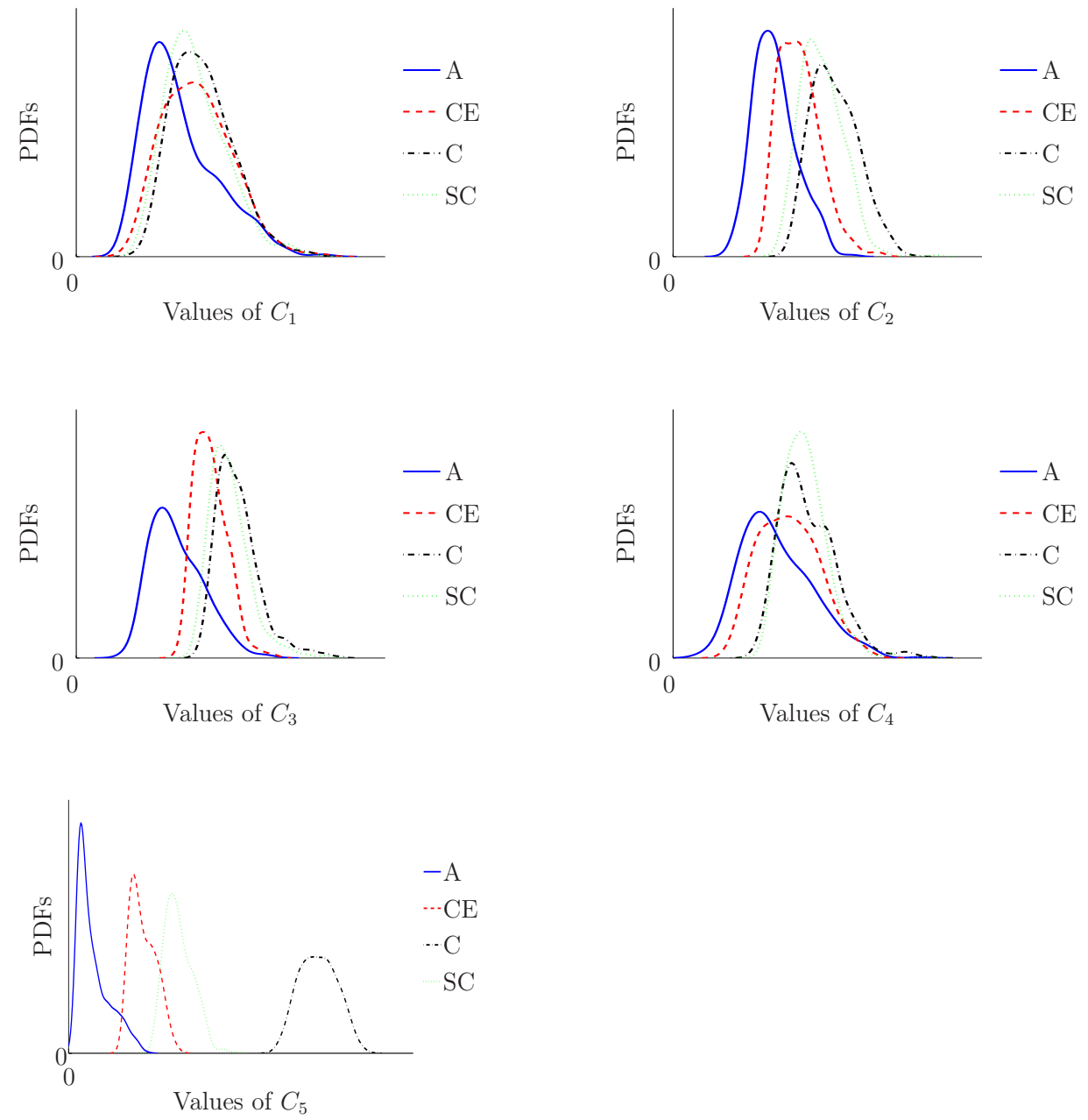

Figure 10: Influence of the track design on the marginal PDFs of vector $\boldsymbol{C}$. 

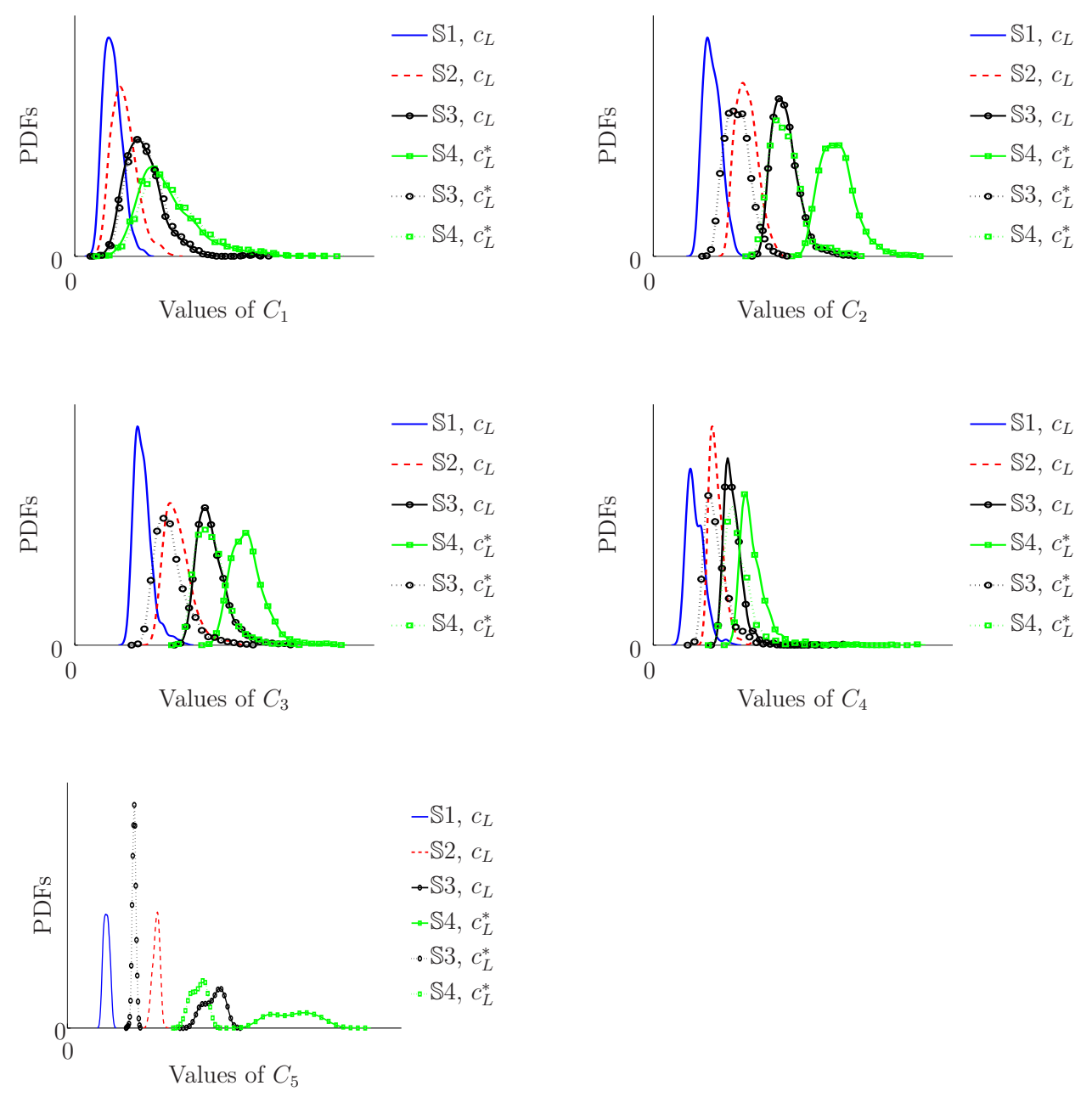

Figure 11: Influence of the train speed on the marginal PDFs of vector $\boldsymbol{C}$. 

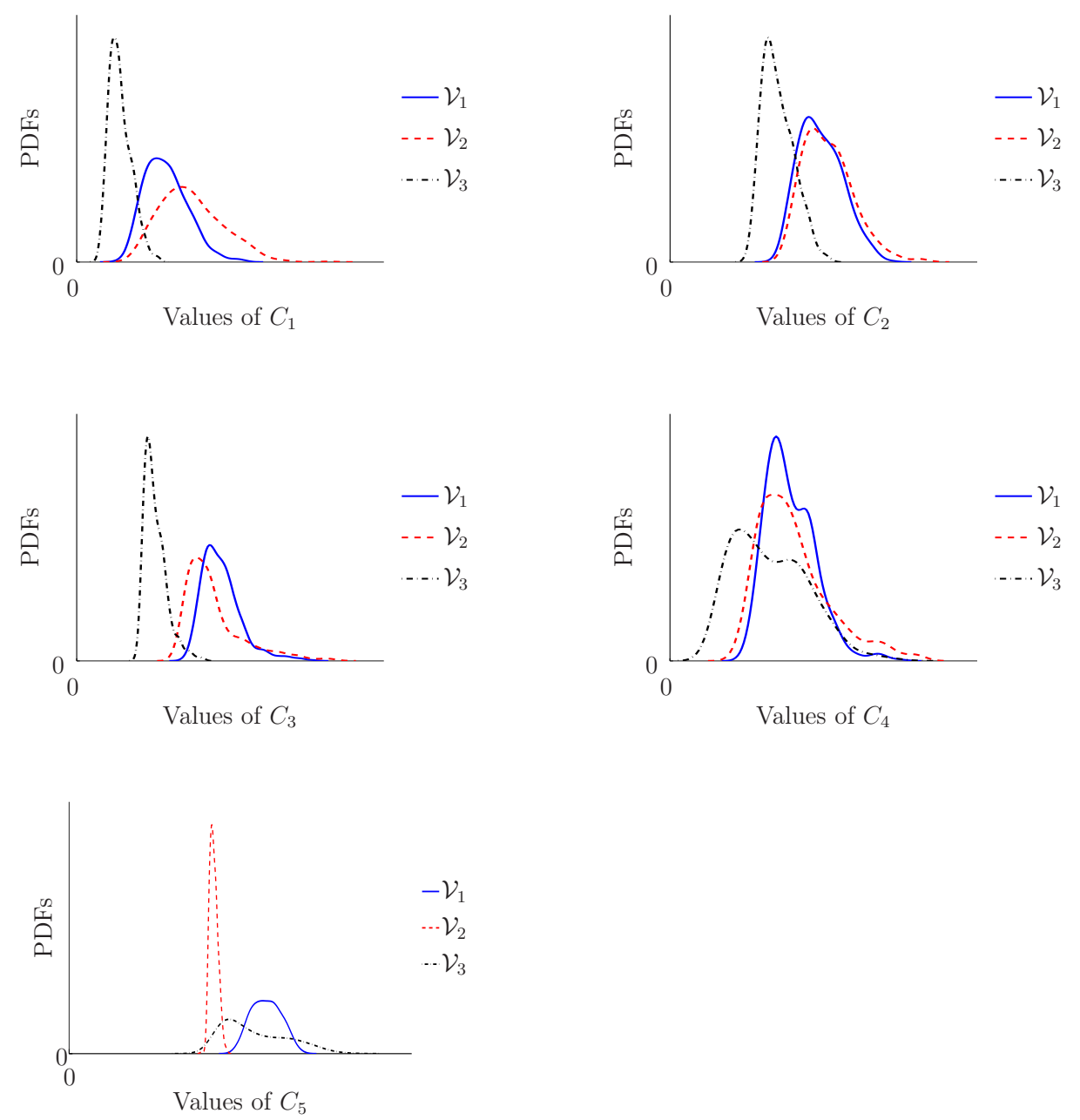

Figure 12: Influence of the train characteristics on the PDFs of $\boldsymbol{C}$. 
the complex link between the track geometry variability and the train dynamics and stability. In particular, three applications have been described in this paper. First, the compared influence of the track design and of the track irregularities has been analyzed. Then, the impact of an increase of the speed on the train stability has been presented. At last, it has been shown to what extent such an approach could be used to compare competitive high speed trains with respect to their response on a set of representative track conditions.

[1] C. Soize, Stochastic Models of Uncertainties in Computational Mechanics., American Society of Civil Engineers, Reston, 2013.

[2] A. Dutfoy, Reference Guide, Tutorial, Open TURNS (www.openturns.org), 2013.

[3] C. Funfschilling, G. Perrin, S. Kraft, Propagation of variability in railway dynamic simulations: application to virtual homologation, Vehicle System Dynamics 50 (2012) $245-261$.

[4] J. Ding, Z. Pan, L. Chen, Second order adjoint sensitivity analysis of multibody systems described by differential-algebraic equations, Multibody System Dynamics 18 (4) (2007).

[5] D. Hickey, K. Worden, M. Platten, J. Wright, Higher-order spectra for identification of nonlinear modal coupling, Mechanical Systems and Signal Processing 23 (4) (2009).

[6] S. Iwnicki, Handbook of railway vehicle dynamics, Taylor and Francis, 2000.

[7] G. Kerschen, K. Worden, A. F. Vakakis, J.-C. Golinval, Past, presentand future of nonlinear system identification in structural dynamics, Mechanical Systems and Signal Processing 20 (3) (2006).

[8] L. Li, K. Phoon, S. Quek, Comparison between karhunen-loève expansion and translation-based simulation of non-gaussian processes, Computers and Structures 85 (2007) 264-76.

[9] K. Petsounis, S. Fassois, Parametric time-domain methods for the identification of vibrating structures - a critical comparison and assessment, Mechanical Systems and Signal Processing 15 (6) (2001).

[10] A. A. Shabana, M. Tobaa, H. Sugiyama, K. E. Zaazaa, On the computer formulations of the wheel/rail contact problem, Nonlinear dynamics 40 (2005).

[11] R. Serban, Analytical derivatives for multibody system analysis, University of Pennsylvania, 2007.

[12] W. Sextro, Dynamical contact problems with friction, Springer, 2002.

[13] J. Wringen, Track to Car body vibration transfer for a bogie rail vehicle, KTH Stockholm, 1997. 
[14] S. Kraft, Parameter identification for a TGV model, Ph.D. thesis, Ecole Centrale de Paris, 2012.

[15] R. Y. Rubinstein, D. P. Kroese, Simulation and the Monte Carlo Method, Second Edition, Wiley, 2007.

[16] L. Fryba, Dynamics of railway bridges, T. Telford, 1996.

[17] F. Au, J. Wang, Y. Cheung, Impact study of cable-stayed railway bridges with random rail irregularities, Engineering Structures 24 (2002) 529-541.

[18] M. Majka, M. Hartnett, Dynamic response of bridges to moving trains: A study on effects of random track irregularities and bridge skewness, Computers and Structures 87 (2009) 1233-1252.

[19] R. Fries, B. Coffey, A state-space approach to the synthesis of random vertical and crosslevel rail irregularities, J. Dyn. Sys., Meas., Control 112 (1) (1990) 83-87.

[20] H. Claus, W. Schiehlen, Modeling and simulation of railway bogie structural vibrations, Vehicle System Dynamics 28 (Suppl) (1997) 538-552.

[21] V. Garg, R. Dukkipati, Dynamics of railway vehicle systems, Academic Press, New York, USA, 1984.

[22] S. Das, R. Ghanem, S. Finette, Polynomial chaos representation of spatio-temporal random field from experimental measurements, J. Comput. Phys. 228 (2009) 87268751.

[23] R. Ghanem, P. D. Spanos, Stochastic Finite Elements: A Spectral Approach, rev. ed., Dover Publications, New York, 2003.

[24] R. Ghanem, A. Doostan, On the construction and analysis of stochastic models: Characterization and propagation of the errors associated with limited data, J. Comput. Phys. 217 (2006) 63-81.

[25] O. Le Maître, O. Knio, Spectral Methods for Uncertainty Quantification, Springer, 2010.

[26] A. Nouy, O. L. Maître, Generalized spectral decomposition method for stochastic nonlinear problems, J. Comput. Phys. 228 (1) (2009) 202-235.

[27] G. Perrin, C. Soize, D. Duhamel, C. Funfschilling, Identification of polynomial chaos representations in high dimension from a set of realizations, SIAM J. Sci. Comput. 34(6) (2012) 2917-2945.

[28] G. Perrin, C. Soize, D. Duhamel, C. Funfschilling, Karhunen-loève expansion revisited for vector-valued random fields: scaling, errors and optimal basis, Journal of Computational Physics 242 (2013) 607-622. 
[29] G. Perrin, C. Soize, D. Duhamel, C. Funfschilling, A posteriori error and optimal reduced basis for stochastic processes defined by a finite set of realizations, SIAM/ASA Journal on Uncertainty quantification (submitted in 2013).

[30] C. Soize, Identification of high-dimension polynomial chaos expansions with random coefficients for non-gausian tensor-valued random fields using partial and limited experimental data., Computer Methods in Applied Mechanics and Engineering 199 (2010) 2150-2164.

[31] G. Perrin, C. Soize, D. Duhamel, C. Funfschilling, Track irregularities stochastic modeling, Probabilistic Engineering Mechanics 34 (2013) 123-130.

[32] H. Hertz, Ueber die beruhrung fester elasticher körper, Journal für reine und angewandte Mathematik 92 (1882).

[33] J. Kalker, Three-Dimensional elastic bodies in rolling contact, KLUWER ACADEMIC PUBLISHER, 1990.

[34] J. Evans, Rail vehicle dynamic simulation using vampire, Vehicle System Dynamics Supplement 31 (1999) 119-140.

[35] O. Evans, Vampire User guide, AEA Technology, 2006. 